Regular Paper

\title{
Influence of Shape and Arrangement of Permanent Magnets on Characteristics of Outer-Rotor IPM Motor
}

\author{
Tadashi YAMAGUCHI ${ }^{* 1}$ (Mem.), Yoshihiro KAWASE ${ }^{* 1}$ (Mem.), Koshiro MATSUDA ${ }^{* 1}$ and Keiichi MORINAGA ${ }^{* 2}$
}

In this paper, we analyzed the magnetic field of an outer-rotor IPM motor using the three-dimensional parallel finite element method in order to investigate the influence of the shape and the arrangement of the permanent magnets. We clarified that providing a flux barrier between the divided magnets is able to reduce the leakage magnetic flux between the divided magnets and to prevent the torque from decreasing. Furthermore, it was clarified that the torque ripple can be reduced while maintaining the average torque by changing the arrangement of the magnets.

Keywords: 3-D parallel finite element method, outer-rotor, IPM motor, magnet arrangement, flux barrier. (Received: 20 July 2018, Revised: 13 December 2018)

\section{Introduction}

Interior permanent magnet (IPM) motors are widely used in electric vehicles, compressor of air conditioners, and the like. The torque characteristics and the electrical loss of the IPM motor strongly depend on the shape and the arrangement of the PMs and the magnetization direction.

IPM motors have a structure in which the permanent magnets are buried in the rotor. In outer-rotor motors, a cylindrical rotor rotates outside the stator. An advantage of the outer-rotor IPM motor is that (i) the magnetic pole can be made up of several PMs in the rotor and (ii) its arrangement can be easily changed.

In this paper, we analyzed several different shapes and arrangements of PMs in which an outer-rotor IPM motor. Consequently, we clarified that the influence of the shape and the arrangement of PMs on the various characteristics of outer-rotor IPM motor.

\section{Analysis Method}

\subsection{Magnetic Field Analysis}

The fundamental equations of the magnetic field considering the eddy current can be written using the magnetic vector potential $\boldsymbol{A}$ as follows:

$$
\begin{aligned}
& \operatorname{rot}(v \operatorname{rot} \boldsymbol{A})=\boldsymbol{J}_{0}+\boldsymbol{J}_{e}+v_{0} \operatorname{rot} \boldsymbol{M} \\
& \boldsymbol{J}_{e}=-\sigma \frac{\partial \boldsymbol{A}}{\partial t}
\end{aligned}
$$

where $v$ is the reluctivity, $\boldsymbol{J}_{0}$ is the exciting current density, $\boldsymbol{J}_{e}$ is the eddy current density, $v_{0}$ is the reluctivi-

\footnotetext{
Correspondence: T.YAMAGUCHI, Department of Electrical Electronic and Computer Engineering, Gifu University, 1-1 Yanagido, Gifu 501-1193, Japan

email: yamachu@gifu-u.ac.jp

${ }^{* 1}$ Gifu University ${ }^{* 2}$ Toyo Denki Seizo K.K.
}

ty of air, $\boldsymbol{M}$ is the magnetization of permanent magnet, and $\sigma$ is the electric conductivity.

\subsection{Electrical Loss Calculation}

The eddy current loss of permanent magnets was calculated by the eddy current density obtained by the magnetic field analysis. The eddy current loss $W_{e d}$ can be written using the eddy current density $\boldsymbol{J}_{e}$ as follows:

$$
W_{e d}=\frac{1}{T} \int_{0}^{T}\left\{\int_{V_{m}} \frac{\left(J_{e}\right)^{2}}{\sigma} d v\right\} d t
$$

where $T$ is the period of the eddy current waveform, $V_{m}$ is the region of the permanent magnet, $\boldsymbol{J}_{e}$ is the eddy current density, and $\sigma$ is the conductivity.

The hysteresis loss and the eddy current loss generated in the steel sheet are approximately estimated from the calculated flux density $\boldsymbol{B}[2]$.

\section{Influence of PMs Shape and Flux Barrier}

\subsection{Analyzed Model and Analysis Condition}

In this section, we investigate the influence of PMs shape and flux barrier on characteristics of outer-rotor IPM motor.

Fig. 1 shows the analyzed model of an outer-rotor IPM motor. The analyzed region is $1 / 2$ in the circumferential direction and is $1 / 2$ in the axial direction of whole region because of the periodicity and the symmetry. This motor has 20 poles and 24 slots, and the coil is the concentrated winding.

Fig. 2 shows divisions of PM. In this analysis, the case where the PMs are not divided (Fig. 2(a)) and the case where the PMs of the respective poles are divided so that the magnetic poles are oriented toward the center of rotation (Figs. 2(b) - 2(e)). The divided PMs are arranged on an arc along the rotor surface so that the magnetic flux towards the stator increases. 
Fig. 3 shows the enlarged view of PM (only 2divisions). The flux barriers are set up between the divided PMs of the division model shown in Fig. 2.

Then, we investigate the influence of PMs shape and flux barrier on various characteristics. Fig. 4 shows the
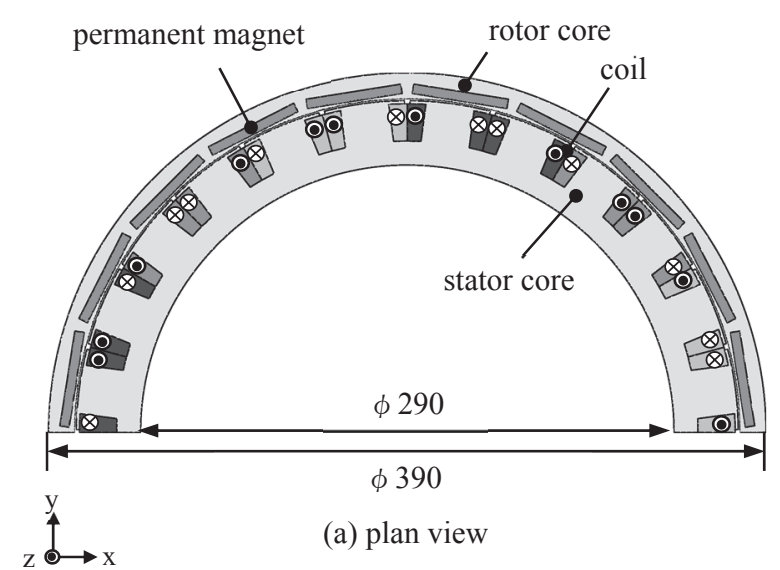

(a) plan view

unit (mm)

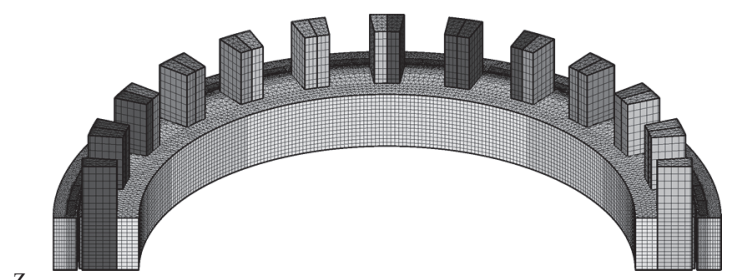<smiles>C[Al]1C=C[Al]1C</smiles>

(b) birds-eye view.

Fig. 1 Analyzed model.

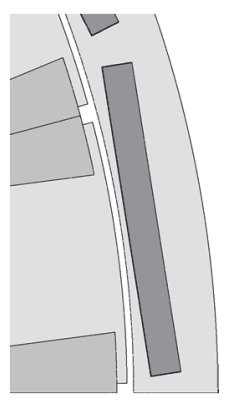

(a) without division

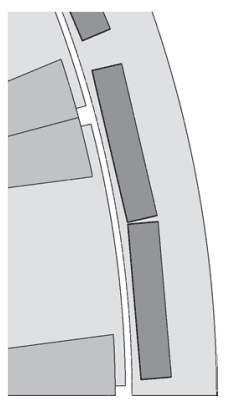

(b) 2-division

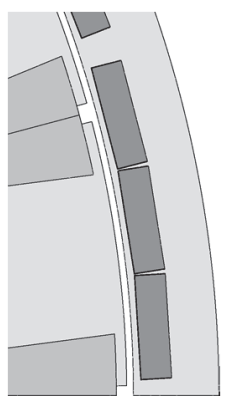

(c) 3-division

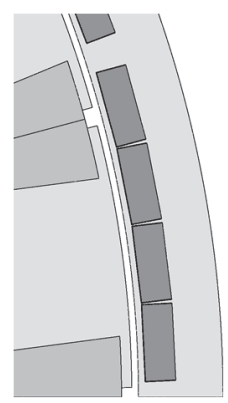

(d) 4-division

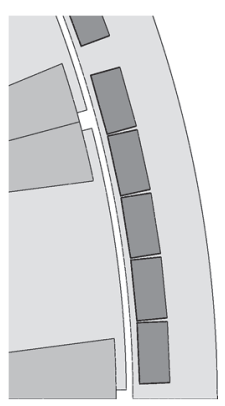

(e) 5-division

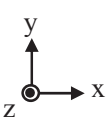

Fig. 2 Divisions of PM.
BH curve. We consider the magnetic nonlinearity of the rotor and stator cores.

Table 1 shows the specification of the motor. In this study, a three phase AC current is applied to the coil, and the steady state analysis is performed.

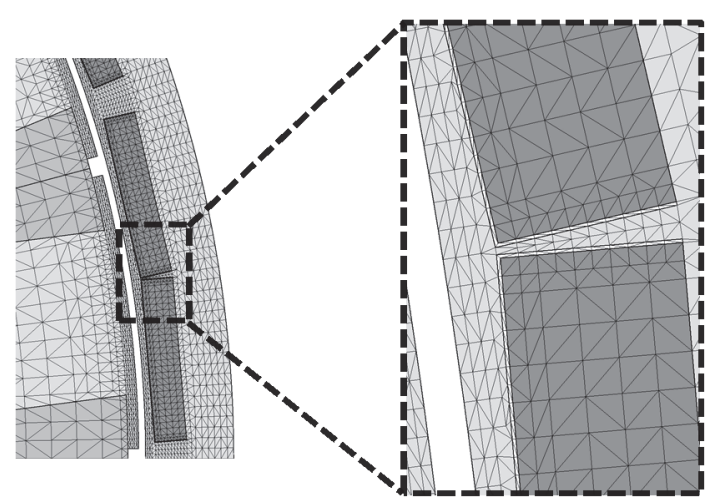

(a) without flux barrier

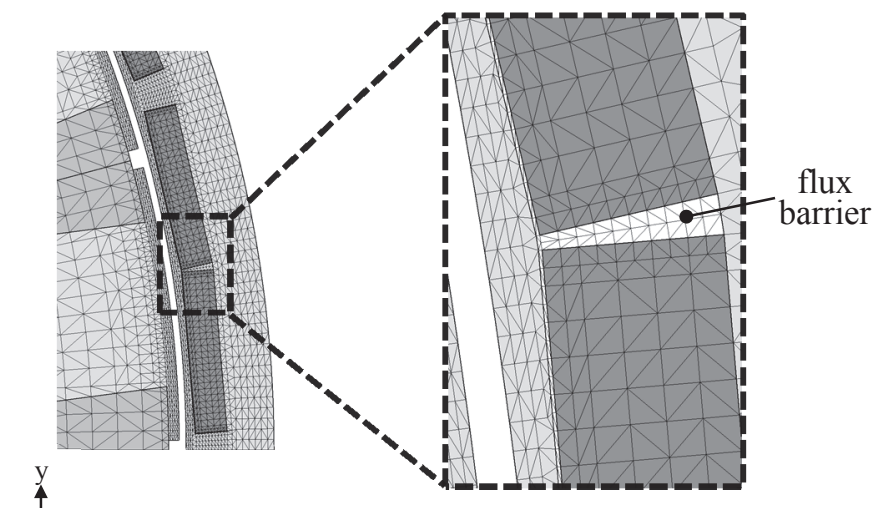

(b) with flux barrier

Fig. 3 Enlarged view of PM (only 2-divison).

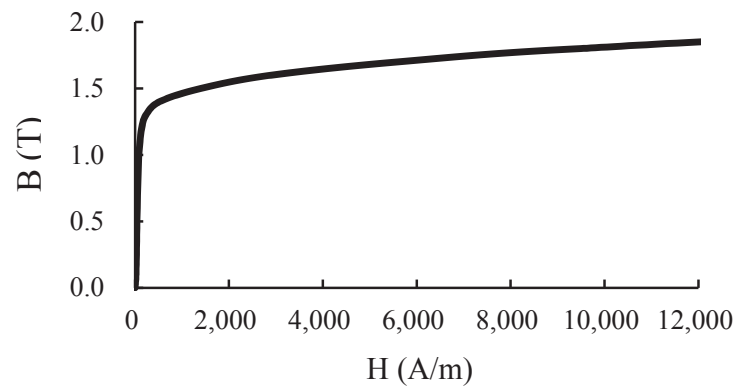

Fig. 4 B-H curve (20HX1200).

Table 1 Analysis conditions

\begin{tabular}{c|c}
\hline Rotation speed (rpm) & 360 \\
\hline Frequency of line voltage $(\mathrm{Hz})$ & 60 \\
\hline Phase current $\left(\mathrm{A}_{\mathrm{rms}}\right)$ & 42 \\
\hline $\begin{array}{c}\text { Conductivity }(\mathrm{S} / \mathrm{m}) \\
\text { Magnetization of permanent } \\
\text { magnet }(\mathrm{T})\end{array}$ & $714,285.7$ \\
\hline Connection & Y connection \\
\hline
\end{tabular}




\subsection{Calculated Results and Discussion}

Fig. 5 shows the distributions of flux density vectors. We can see that the magnetic flux of the stator in the divided PM models is larger than that of the withoutdivision model. This is because the magnetic poles come close to the air gap and the magnetic flux towards the stator increases. We can also see that the leakage magnetic flux between the divided PMs increases as the number of PM divisions increases when there is not the flux barrier between the divided PMs. On the other hand, we can see that the leakage magnetic flux between the divided PMs is reduced when there is the flux barrier between the divided PMs.

Fig. 6 shows the torque waveforms. Those values are normalized by the average torque of the IPM motor

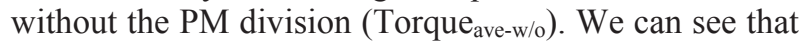
the torque of the divided PM models is larger than that of the without-division model. This is because the magnetic poles come close to the air gap and the magnetic flux towards the stator increases. We can also see that the torque when there is the flux barrier is larger than that when there is not the flux barrier.

Fig. 7 shows the average torque. We can see that the average torque decreases as the number of PM divisions increases when there is not the flux barrier between the divided PMs. This is probably because that the leakage magnetic flux between the divided PMs increases as the number of PM divisions increases. In addition, we can see that the average torque does not decrease greatly even if the number of PM divisions increases when there is the flux barrier between the divided PMs. This is probably because that the leakage magnetic flux between the divided PMs is reduced.

Fig. 8 shows the electrical loss characteristics. Those values are normalized by the sum of the electrical loss of the IPM motor without the PM division (Loss sum-w/o $_{\text {) }}$. We can see that the eddy current loss of the PMs decreases as the number of PM divisions increases. In addition, we can see that the eddy current loss of the PMs is similarly reduced even if there is the flux barrier between the divided PMs. This is because the leakage magnetic flux between the divided PMs is reduced by setting up the flux barrier between the divided PMs, however, the direction and magnitude of the magnetic flux are stable. Moreover, we can see that the sum of the electrical loss has not changed significantly regardless of the presence or absence of the flux barrier.

\section{Influence of Shape and Arrangement of PMs}

\subsection{Analyzed Model and Analysis Condition}

In this section, we investigate the influence of shape and arrangement of PMs on the characteristics of the outer-rotor IPM motor.

We analyzed the outer-rotor IPM motor with four patterns of the shape and the arrangement of PMs as shown in Fig. 9. Fig. 9(a) is the conventional model. Magnetization directions of Figs. 9(b) - 9(d) are differ- ent from that of Fig. 9(a), and the number of divisions of the PMs is different from each other.

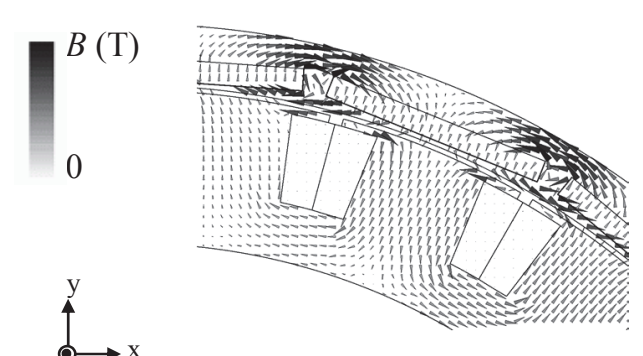

(a) without division

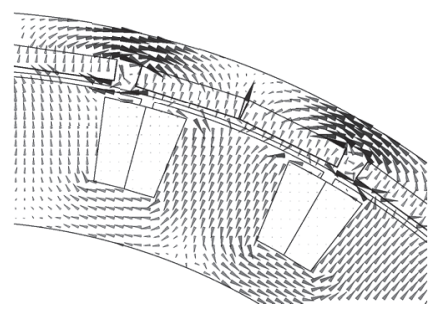

(i) without flux barrier

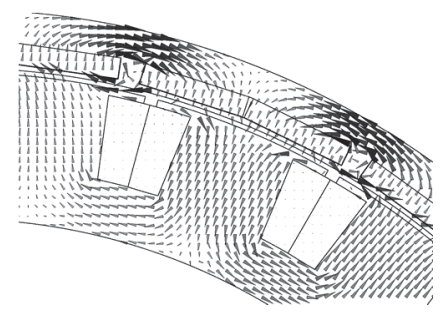

(ii) with flux barrier (b) 2-division

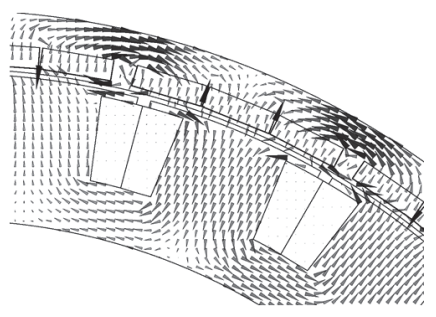

(i) without flux barrier

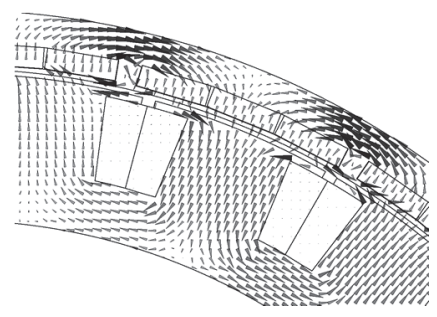

(ii) with flux barrier (c) 3-division

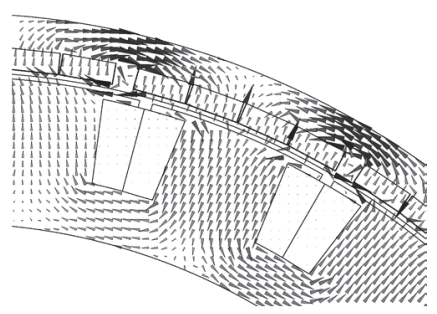

(i) without flux barrier

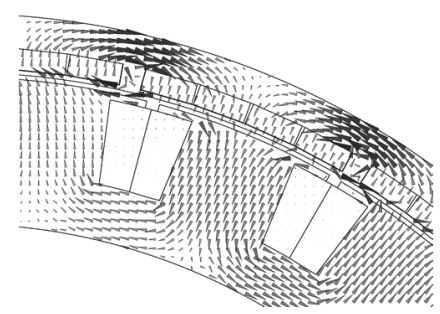

(ii) with flux barrier (d) 4-division

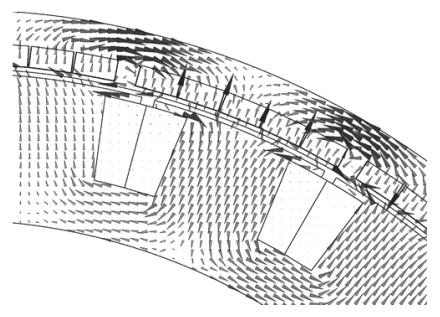

(i) without flux

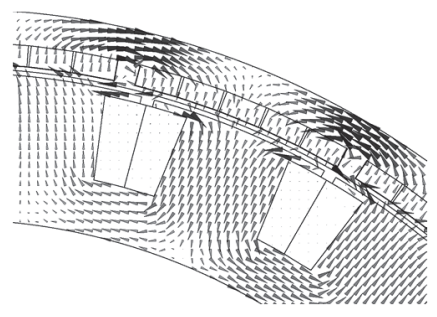

(ii) with flux barrier (e) 5-division

Fig. 5 Distributions of flux density vectors. 


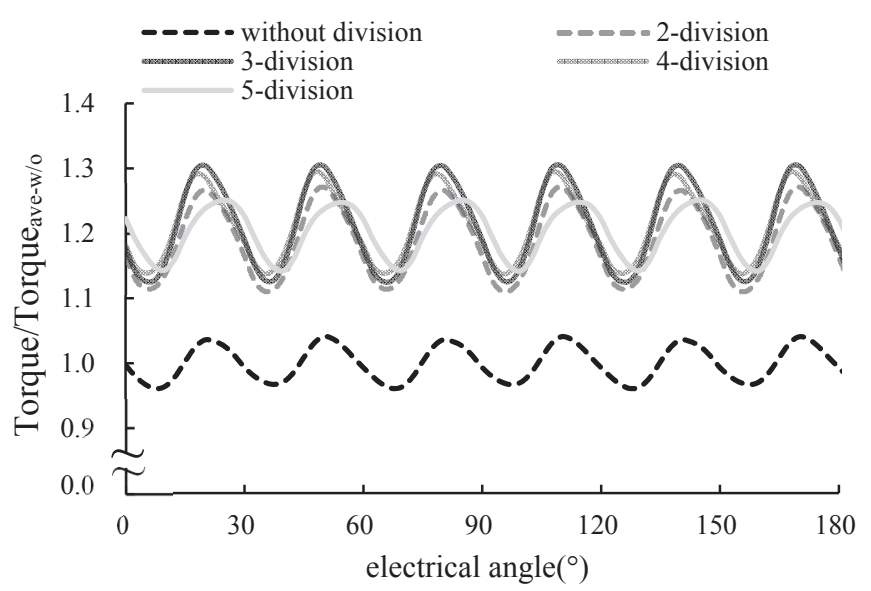

(a) without flux barrier
The other analysis conditions are the same as those in Section 3.

\subsection{Calculated Results and Discussion}

Fig. 10 shows the distributions of flux density vectors. We can see that parts of magnetic flux saturation in Figs. 10(b)-(d) are different from those in Fig. 10(a). The distance between the magnetic poles is short in the conventional model so that the magnetic flux is shortcircuited between the magnetic poles. On the other hand, in the PM arranged model, the distance between magnetic poles becomes large so that the magnetic flux becomes difficult to short-circuit. Fig. 11 shows the induced voltage waveforms of the U-phase. Those values are normalized by the maximum voltage of the

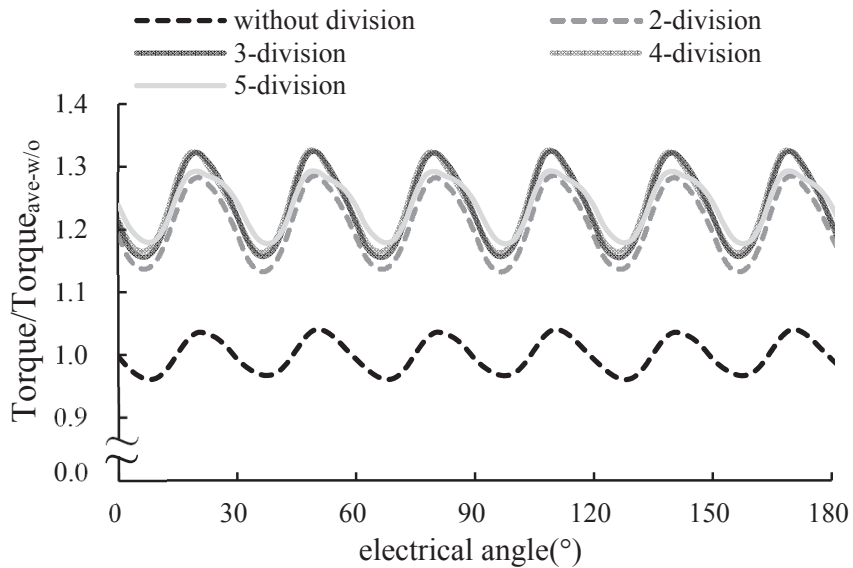

(b) with flux barrier

Fig. 6 Torque waveforms.

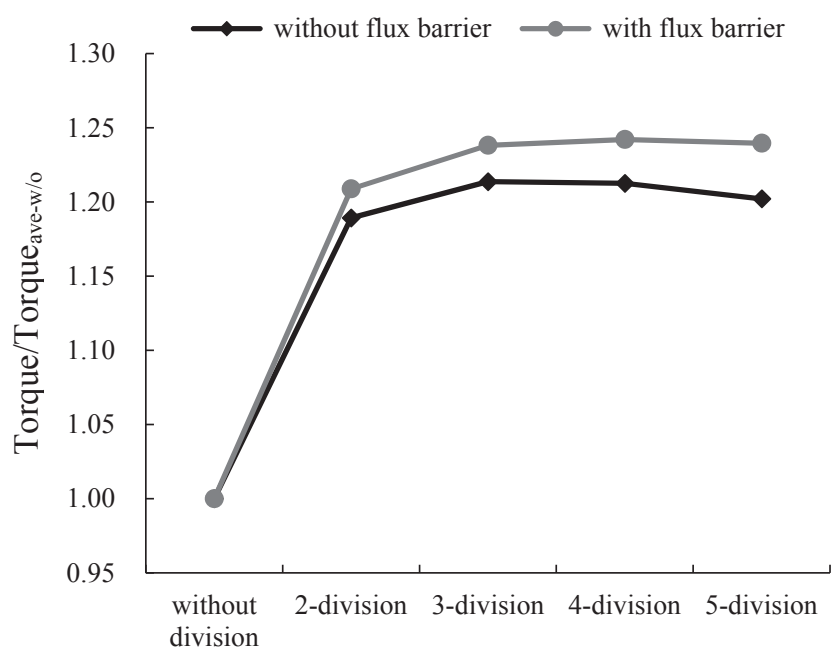

Fig. 7 Average torque.

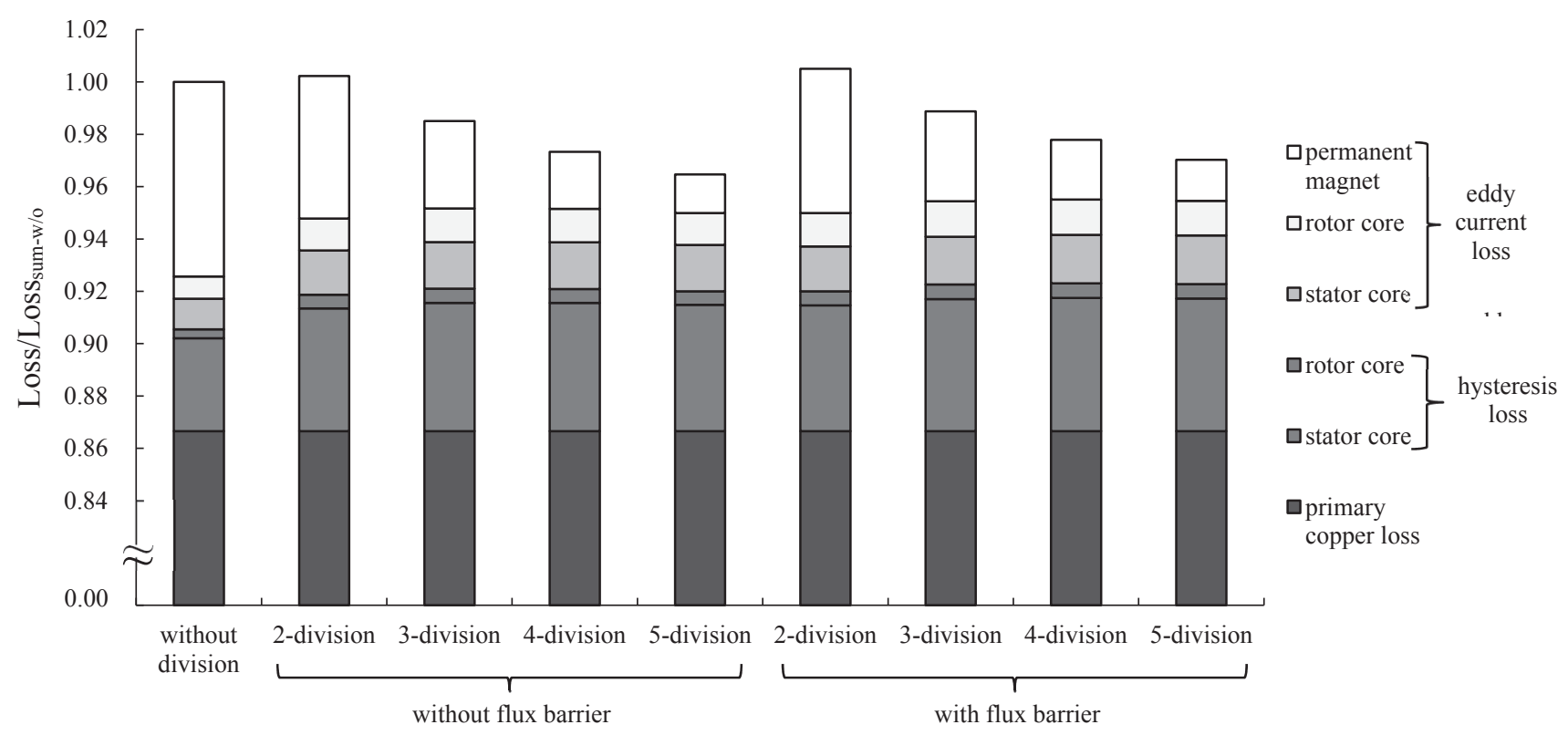

Fig. 8 Electrical loss characteristics. 


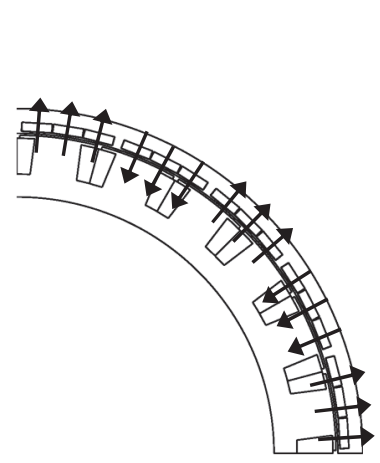

(a) conventional

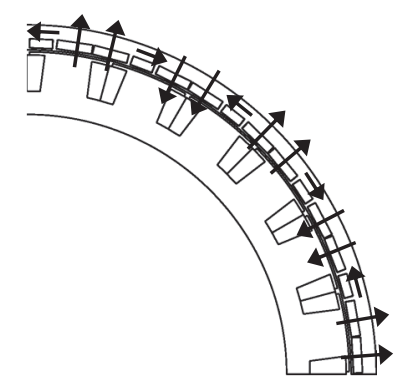

(c) 2-division

\section{magnetization direction}

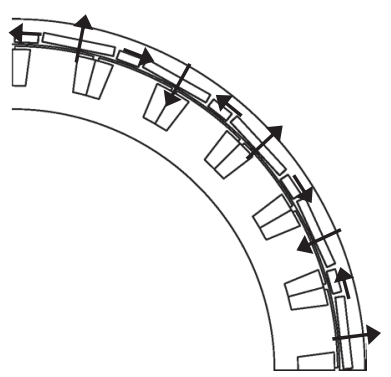

(b) without division

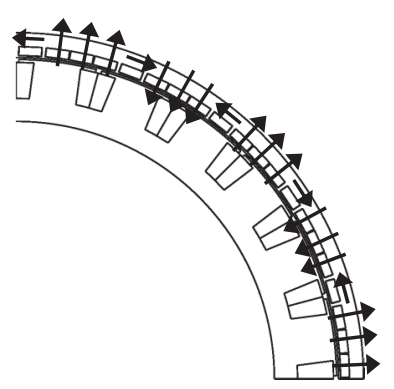

(d) 3-division

Fig. 9 Shape and arrangement of PMs.

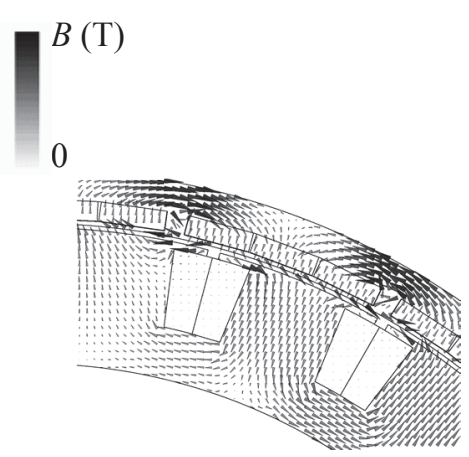

(a) conventional

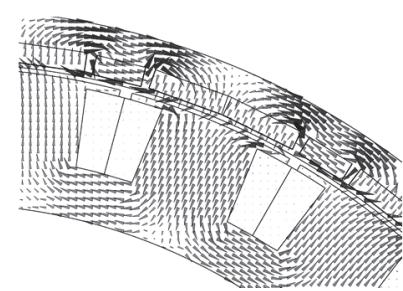

$\stackrel{\mathrm{y}}{\mathrm{z}} \mathrm{x}$

(c) 2-division

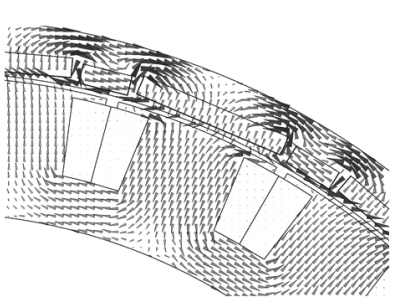

(b) without division

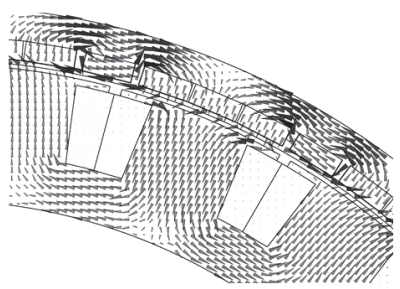

(d) 3-division

Fig. 10 Distributions of flux density vectors.

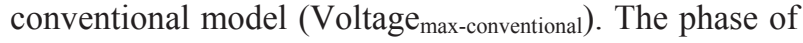
the induced voltage waveform of the conventional model is different from those of the other models because the initial position of the rotor are different each other.

Fig. 12 shows the total harmonic distortion (THD) of the induced voltage of the U-phase. Those are obtained using the following equation:

$$
\mathrm{THD}=\frac{\sqrt{\sum_{n \geq 2} V_{n}^{2}}}{V_{1}} \times 100(\%)
$$

where $V_{n}$ is the effective value of the $n$-th harmonic voltage.

We can see that the THD of the induced voltage is small in the other models as compared with the conventional model. This is because the magnetic flux shortcircuited between the magnetic poles is reduced and the distortion of the magnetic flux density distribution in the air gap is also reduced.

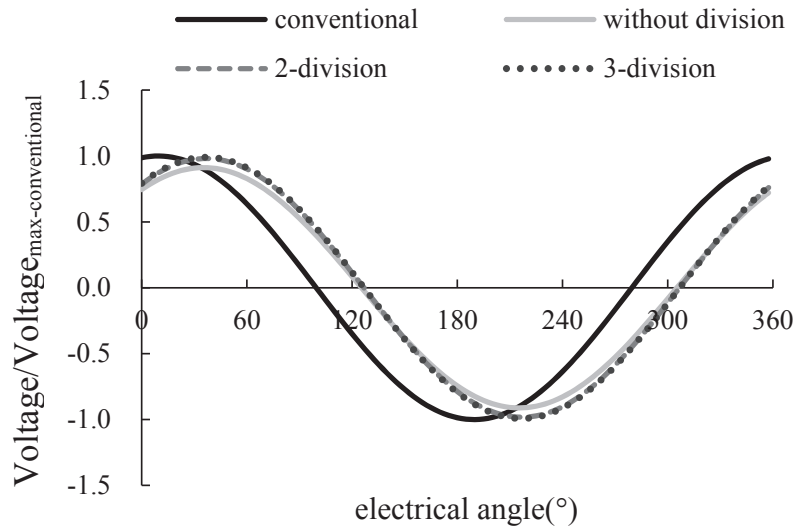

Fig. 11 Induced voltage waveforms of U-phase.

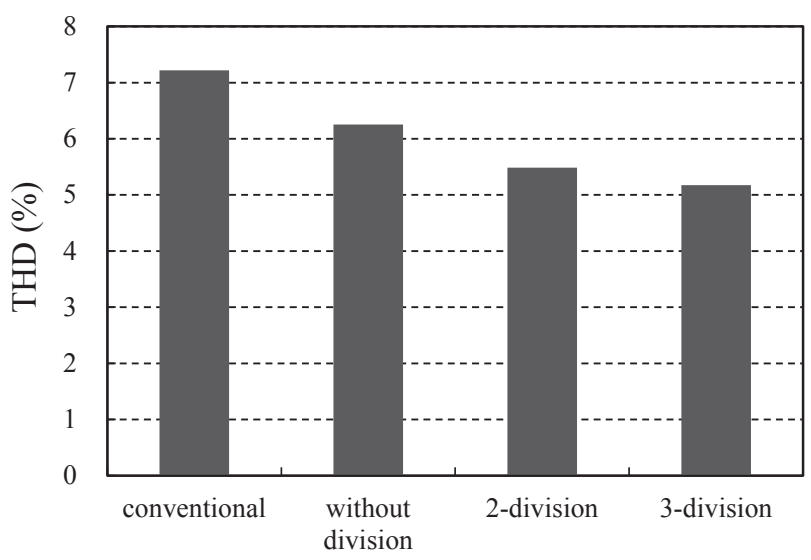

Fig. 12 THD of induced voltage of U-phase. 


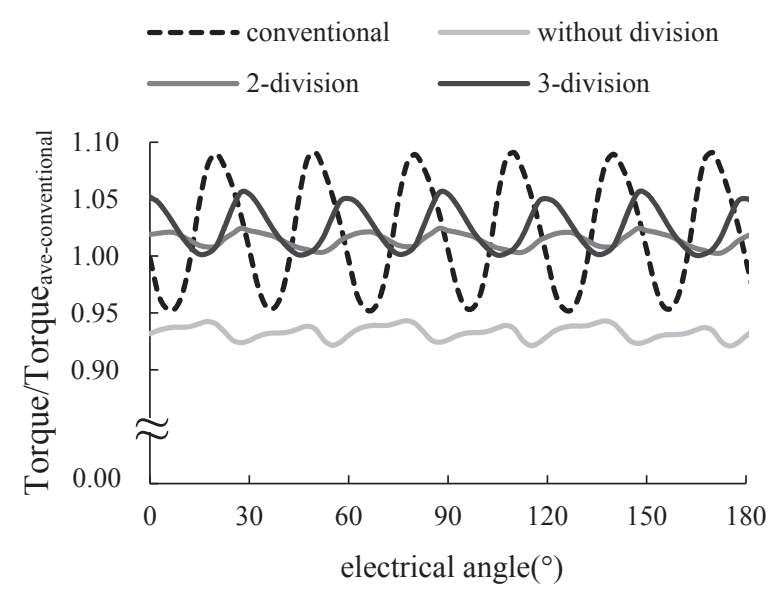

Fig. 13 Torque waveforms.

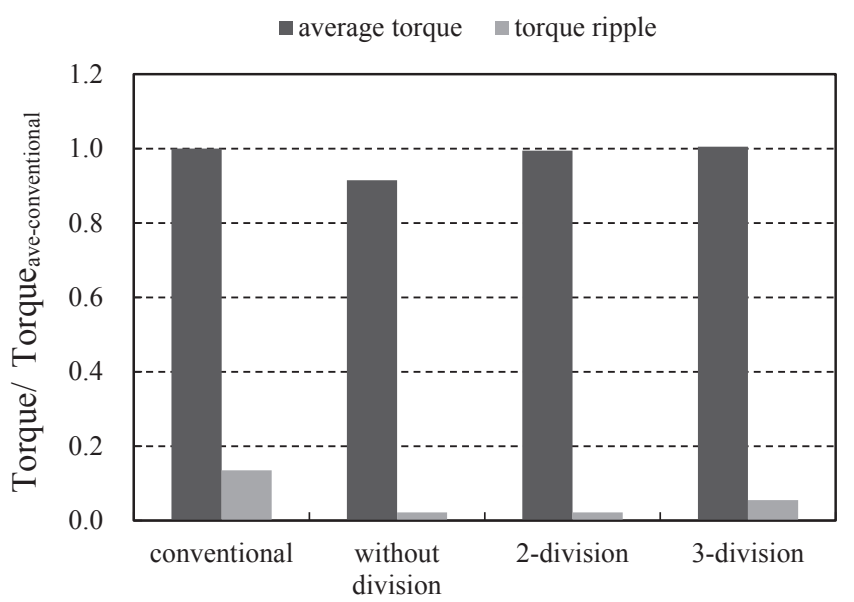

Fig. 14 Torque characteristics.

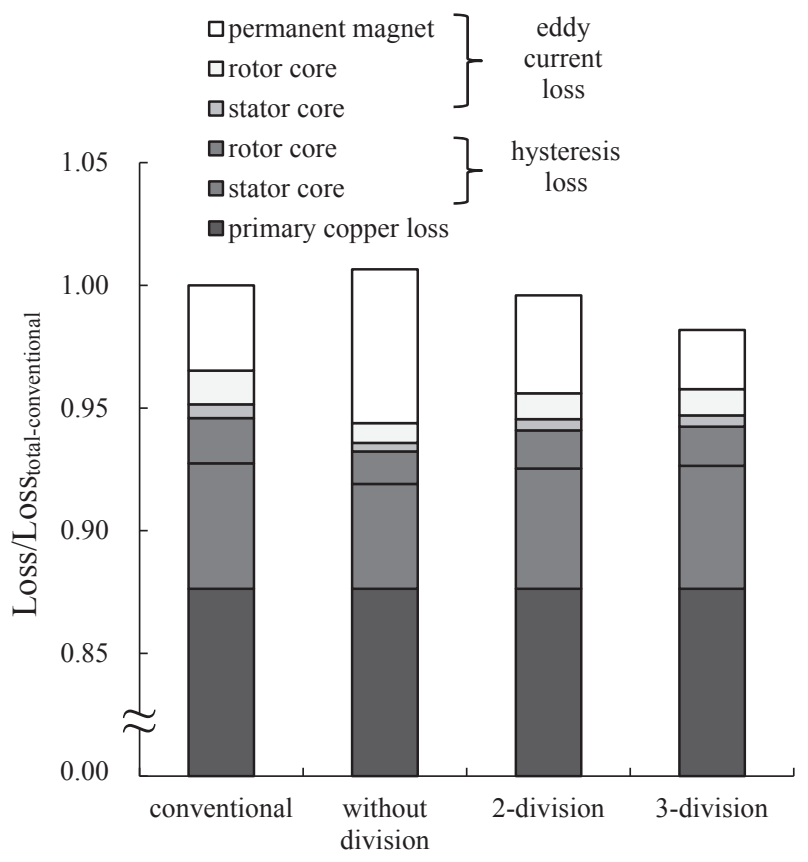

Fig. 15 Electrical loss characteristics.
Figs. 13 and 14 show the torque waveforms and the torque characteristics, respectively. Those values are normalized by the average torque of the conventional model (Torque ${ }_{\text {ave-conventional }}$. We can see that the torque ripples of the 2-division model and the 3-division model are small while those average torques are equal to that of the conventional model. The distortion of the magnetic flux density distribution in the air gap decreases so that the magnetic flux distribution comes close to a sinusoidal wave and the torque ripple decreases. This is also confirmed from the fact that the distortion of the induced voltage is reduced in Fig. 12.

Fig. 15 shows the electrical loss characteristics. Those values are normalized by the sum of the electrical

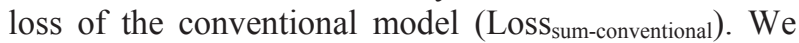
can see that the eddy current loss of the PMs of the without-division model is larger than that of the conventional model. This is because the length of the PMs of the without-division model is larger than that of the conventional model. The eddy current loss is reduced by dividing the PMs into two and three. We can also see that there is a little difference in the sum of the electrical loss in any model.

From Figs. 14 and 15, we can see that the average torque increases and the sum of the electrical loss decreases by dividing the PMs. That means that the efficiency of the motor is improved by dividing the PMs.

\section{Conclusion}

In this paper, we analyzed the magnetic field of an outer-rotor IPM motor using the three-dimensional parallel finite element method in order to investigate the influence of the shape and the arrangement of the permanent magnets.

As a result, the following knowledge was obtained:

- The leakage magnetic flux between the divided PMs increases and the torque decreases as the number of PM divisions increases. However, it is possible to reduce the leakage magnetic flux between the divided PMs and prevent the torque from decreasing by setting up the flux barrier between the divided PMs.

- The torque ripples of the models in which the permanent magnets are devided into two or three are small while the average torques are equal to that of the conventional model. Moreover, there is a little difference in the sum of the electrical loss in any model.

\section{References}

[1] S. Ito and Y. Kawase, "Computer Aided Engineering of Electric and Electronic Apparatus Using Finite Element Method", Morikita Publishing Co, 2000.

[2] K. Yamazaki, "Loss Calculation of Induction Motors Considering Harmonic Electromagnetic Field in Stator and Rotor", IEEJ Trans. on IA, vol.123, no.4, pp.392-400, 2003. (in Japanese) 